\title{
Anti-Aliasing, Anti-Leakage Fourier Transform
}

Michel Schonewille, Andreas Klaedtke and Alan Vigner (PGS)

\section{SUMMARY}

The anti-leakage Fourier transform (ALFT) is a robust and easy to implement regularisation method. In this talk first several aspects of the ALFT are illustrated using simple synthetic signals. The key observations are: 1) When the Fourier spectrum is sparse, very good results can be obtained using the ALFT with a limited number of iterations; 2) When the spectrum is not sparse, but data are well sampled, a very large number of iterations can be required for a similar quality reconstruction of the data as with Least Squares Fourier regularisation; 3) Sparsely sampled data with a sparse spectrum can be handled by the ALFT if the sampling is irregular, but results strongly degrade if the sampling is (close to) regular.

To improve the handling of sparsely sampled data it is proposed to derive a weighting function in the spatial Fourier domain from the lower temporal frequencies. The weighting function is extrapolated to the higher frequencies where the data may be spatially aliased, and instead of selecting the strongest Fourier component in each iteration (as done in the standard ALFT), the component that would be strongest after weighting is selected. The advantages are shown on synthetic and field data. 